\title{
CONSIDERACIONES EN PATOLOGÍA NO INVASIVA
}

\section{Hiperplasia epitelial y enfermedad proliferativa}

Esta clasificación se basa en estudios epidemiológicos de seguimiento que intentan establecer un nexo entre los patrones histológicos y el grado de riesgo de cáncer mamario.

En lugar de suponer una progresión gradual en un espectro continuo desde la ausencia de cambios hasta carcinoma in situ, el enfoque se debe orientar hacia una separación clara del carcinoma in situ y otros patrones de hiperplasia que se describen a continuación:

Adenosis: se define como el aumento de elementos glandulares sin un incremento concomitante en la relación con la membrana basal (no hay más de dos capas celulares a partir de ésta). En la presentación Florida, hay una mayor actividad epitelial con proliferación de acinos; es más frecuente en jóvenes y no se asocia con cáncer.

Adenosis esclerosante: la proliferación epitelial aumenta y con el tiempo puede producir áreas de fibrosis conformando nodulaciones que disminuyen en la menopausia; se presenta en mujeres mayores de 30 años.

Hiperplasia mamaria: al igual que las otras hiperplasias, se considera como el aumento de la cantidad de células en relación con la membrana basal.

Normalmente se observan dos capas de células por encima de la membrana basal, por lo tanto para que se pueda denominar hiperplasia deben observarse tres o más capas celulares. Hasta cuatro capas se denomina hiperplasia leve, cuando alcanza cinco o más capas se denomina hiperplasia moderada e hiperplasia florida. Estas últimas tienen tendencia a atravesar y distender los espacios donde se producen, asignándose la categoria de florida cuando las anormalidades son más pronunciadas, pero en verdad no existe un criterio estricto para asignarlas a un grupo u otro. Este tipo de hallazgos se encuentra en el $\mathbf{2 0 \%}$ de las biopsias.

Tipos de hiperplasia: lobulillar, apocrina y habitual o ductal. Esta última puede tener orígen en el interior de los acinos. Parece razonable evitar el nombre de ductal.

Hiperplasia Ductal Atípica: En ésta las anomalías comienzan a semejarse a los patrones observados en el carcinoma in situ. Mientras que las hiperplasias leve y moderada se diferencian por la cantidad de capas celulares, las lesiones severas y la hiperplasia ductal atípica se diferencian por rasgos cualitativos de patrones intercelulares y citológicos. El manejo de estas lesiones es básicamente la observación y seguimiento de acuerdo con los factores de riesgo.

\section{Pronóstico}

Las diferentes lesiones pueden ser clasificadas de acuerdo con el riesgo potencial de presentar un carcinoma como de riesgo leve, moderado y pronunciado, donde leve indica un riesgo de 1.5 a 2 veces mayor que la población general, y pronunciado indica un riesgo aproximado de 10 veces. La correlación entre estos parámetros y el riesgo de cáncer se encuentra ilustrada en el siguiente cuadro:

\author{
RIESGO RELATIVO DE DESARROLLAR \\ CANCER DE MAMA
}

- Sin riesgo (ausencia de enfermedad proliferativa)

Cambio apocrino

Ectasia de los conductos

Hiperplasia epitelial leve de tipo habitual.

- Riesgo ligeramente aumentado (1.5 - 2 veces)

Hiperplasia de tipo habitual moderada o florida

Adenosis esclerosante

Papiloma

- Riesgo moderadamente aumentado (4 - 5 veces)

Hiperplasia atípica o lesiones fronterizas

-Hiperplasia ductal atípica

-Hiperplasia lobulillar atípica

- Alto riesgo ( 8 - 10 veces)

Carcinoma ductal in situ no comedo

Carcinoma lobulillar in situ

Esta clasificación no representa una nomenclatura universal; más bien son el resultado de trabajos que intentaron limitar esta denominación a un pequeño grupo de patrones histológicos con rasgos análogos a los del CA IN SITU

\section{Otras condiciones mamarias benignas}

Macroquistes: cerca del $76 \%$ se presentan en la premenopausia y su mayor frecuencia ocurre entre los 35 y los 50 anos. Pueden coexistir con adenosis y fibrosis y algunos autores los asocian con secreción activa del epitelio apocrino bajo estímulo estrogénico.

Ectasia Ductal Canalicular: por algunos denominada comedomastitis, consiste en la dilatación de los conductos mamarios mayores e intermedios por debajo del pezón y la areola. En algunos casos se acompana de fibrosis e inflamación que pueden producir retracciones, conformar masa o fistulizarse y presentar infección secundaria. Frecuentemente se encuentra en reportes mamográficos y no se ha asociado con riesgo de cáncer. Cuando hay recidivas frecuentes puedes ser necesaria la resección de conductos terminales.

Necrosis grasa: es una tumoración firme difícil de diferenciar clínicamente del carcinoma. Es secundaria en algunos casos a trauma, y la mayoría de veces espontánea; en casos avanzados puede producir fibrosis con retracción de la piel. Requiere bacaf para confirmación, y ante la duda sedebe practicar tumorectomía amplia.

Fibroadenoma: es el tumor benigno más frecuente de la mama, de mayor frecuencia en pacientes jóvenes. Es una lesión firme, lisa, cauchosa, móvil y usualmente bien definida; en mujeres adultas puede tener calcifica- ciones. No predispone a carcinoma y pueden ser confirmada con bacaf o extirpada con anestesia local.

Papilomatosis Juvenil: se trata de una tumoración indurada constituída por dilatación de los conductos galactóforos y proliferación intraductal, conformando 
múltiples quistes macroscópicos. Ocurre en mújeres menores de 30 años y se trata mediante resección local.

\section{CARCINOMA IN SITU (ESTADO O (TIS NOMO)}

Tumor en proliferación celular epitelial confinado a los ductos mamarios o lobulillos sin traspasar la membrana basal, la mamografía de buena calidad ha permitido aumentar su diagnóstico.

Teniendo en cuenta la biología y la historia natural y el comportamiento, los tipos camalicular y lobulillar deben considerarse entidades diferentes como se describe a continuación:

\section{CARCINOMA LOBULILLAR IN SITU (CLI)}

El término carcinoma lobulillar in situ (CLIS) fue acuñado y sustentado por Foote y Stewart en 1941. Los términos CLIS e hiperplasia lobulillar atípica (HLA) se pueden incluir en la denominación NEOPLASIA LOBULILLAR ATIPICA. Es una circunstancia desafortunada que la categoría carcinoma lobulillar in situ conserve el término «carcinoma», ya que fundamentalmente es solo un indicador de riesgo aumentado, más que un marcador del sitio en-el cual un futuro cáncer de mama se desarrollará. Una mastectomía unilateral es por lo tanto un tratamiento inapropiado y exagerado.

\section{Rasgos del Carcinoma Lobulillar In Situ}

Multifocal, multicéntrico, bilateral

Menor frecuencia después de la menopausia

No palpable, hallazgo incidental incluso en mamografía Indica alto riesgo de carcinoma invasivo ulterior

Riesgo relativo 8 a 11 veces mayor al de la población general

Riesgo absoluto $\mathbf{2 0}$ a $\mathbf{2 5 \%}$ a 15 años después del diagnóstico

Coexistencia con carcinoma infiltrante de tipos especiales (lobulillar y tubular).

\section{Incidencia}

Es difícil de precisar, ya que el diagnóstico generalmente es incidental al obtener muestras por alguna otra indicación. Algunos autores han reportado frecuencias del 2 al $5 \%$, con un pico de incidencia entre los 40 y 46 años; entre el 80 y $90 \%$ de los casos corresponden a mujeres premenopáusicas. Se ha discutido sobre la posibilidad de regresión en la menopausia; Page demostró decrecimiento en 15 anos. Tiene un potencial de riesgo de progresar a carcinoma infiltrante de $1 \%$ por ano. Se trata de una enfermedad sin manifestación clínica ni mamográfica. Tiende a permanecer silenciosa ya que solamente el $25 \%$ evolucionan hacia carcinoma. Por lo tanto muchas mujeres con carcinoma lobulillar in situ no diagnosticado fallecen por otra causa. Es hallazgo incidental en 1 a $2 \%$ de muestras mamarias por lo demás benignas.

La incidencia ha variado por:

1. Implementación más generalizada de la mamografía (hay leve asociación con microcalcificaciones 25-30\%).

2. Mayor tendencia a biopsiar lesiones mamográficamente sospechosas.
3. Mayor conciencia de las lesiones macroscópicamente inaparentes y estudios más complejos y concienzudos por los patólogos en la realización de los cortes histológicos.

Al igual que otras lesiones es más frecuente en el lado izquierdo y afecta las regiones con mayor contenido de tejido mamario (región sub-areolar y cuadrante superior externo).

Tiene mayor tendencia a ser multicéntrico y bilateral. Si la biopsia demuestra CLIS la posibilidad de focos con enfermedad residual en la misma mama es mayor del $50 \%$ y la probabilidad de que los haya en la mama contralateral, si se realizan biopsias en forma amplia, es del 50\%.

La enfermedad invasora coexistente es probable pero cada vez más rara desde el advenimiento de la mamografía; varía del 4 al $6 \%$.

Luego de la detección por biopsia y el tratamiento el riesgo de cáncer invasor es 10 veces mayor que en la población general. El riesgo absoluto es $25-30 \%, 15$ a 20 años después del diagnóstico.

El tipo de enfermedad invasora asociado es generalmente tubular y lobulillar, ambos con un pronóstico algo más favorable que los otros tipos histológicos.

El notable índice de sobrevida registrado ha sido atribuido a un régimen estricto de vigilancia que permite detectar el carcinoma en una fase más temprana.

La coexistencia de carcinoma ductal in situ y lobulillar in situ es del $5-10 \%$.

\section{Manejo del Carcinoma Lobulillar In Situ}

El manejo del carcinoma lobulillar in situ es bastante controversial. Por mucho tiempo se realizó mastectomía simple unilateral con biopsia en espejo contralateral, lo cual no se considera racional hoy en día a la luz del comportamiento biológico de la entidad.

Es necesario tratar la totalidad del tejido mamario, y no cada glándula por separado. Por esto las resecciones unilaterales totales o parciales no tienen ningún valor actualmente.

El tratamiento debe estar dirigido bien sea a:

1. Eliminar totalmente el riesgo de desarrollar un carcinoma infiltrante (situación que solo se lograría con mastectomía total bilateral), ó

2. Buscar disminuir esta posibilidad mediante fármacos que tengan posibilidades de quimioprevención, ó

3. Detectar precozmente un carcinoma mamario invasor mediante el uso sistemático de la mamografía de alta resolución.

\section{Mastectomía Total Bilateral con o sin Reconstrucción}

La mastectomía total bilateral seguida de reconstrucción mamaria inmediata, ya sea con prótesis o rotación de colgajos musculocutáneos, es una solución definitiva aunque para algunos autores es una actitud demasiado drástica ya que aproximadamente un $70 \%$ de estas pacientes nunca padecerán de un CARCINOMA INFILTRANTE. Esta opción deberá restringirse a aquellas que presenten un alto grado de cancerofobia y no se muestren dispuestas a mantener un seguimiento clínico y mamográfico prolongado en espera de la aparición de un tumor infiltrante. Factores que podrían inclinar la balan- 
za hacia este manejo serían la existencia de antecedentes claros de CARCINOMA MAMARIO en pariente de primer grado de consanguinidad o la dificultad para el seguimiento mamográfico por la presencia de tejido fibroglandular denso.

\section{Tamoxifén}

Recientemente el NSABP en cooperación con instituciones canadienses está reclutando 16.000 mujeres aleatorizadas para recibir tamoxifén o placebo con la hipótesis de que este fármaco puede disminuír la incidencia de CARCINOMA INFILTRANTE. En presencia de Receptores de Estrógeno positivos y Her2/neu negativo, cuando es posible practicarlos, el tamoxifén estaría bien indicado. El Instituto de Tumores de Milán está llevando a cabo otro estudio prospectivo con tamoxifén, reclutando pacientes sanas sin riesgo y que hayan sido sometidas a histerectomía. Tendremos que esperar por lo menos hasta el año 2002 años para evaluar los resultados definitivos.

\section{Seguimiento Clínico y Mamográfico}

La alternativa final en el manejo del CARCINOMA LOBULILLAR IN SITU es el seguimiento clínico y mamográfico periódico, en espera de identificar el tumor en fase preclínica, ya sea ductal in situ o infiltrante. Esta actitud requiere de una paciente muy bien informada y dispuesta a correr el riesgo anotado. Tanto el médico como la paciente deben recordar que un carcinoma infiltrante no palpable se asocia hasta en un 20\% de los casos con metástasis ganglionares axilares, lo que ensombrece el pronóstico de este grupo de mujeres.

En el momento actual la mayoría de las pacientes optan por el manejo conservador de seguimiento, reservando la mastectomía profiláctica para aquellas pacientes con otros factores de riesgo concurrentes o aditivos como historia familiar y ansiedad extrema.

El manejo actual del carcinoma lobulillar in situ debe realizarse idealmente dentro de protocolos prospectivos de investigación o mediante junta multidisciplinaria. En caso de que esto no sea posible el médico debe procurar que la paciente participe en la decisión. Siempre deberá tenerse en cuenta además de la edad, el grado de ansiedad que refleje la mujer, las posibilidades de controlarla, los antecedentes familiares y los estudios genéticos si están disponibles.

\section{CARCINOMA DUCTAL IN SITU (CDIS)}

El CDIS es considerado como un verdadero precursor anatómico del carcinoma invasor de mama. Hay varios argumentos que apoyan esta conclusión:

1. La revisión de casos de CDIS tratados con biopsia únicamente muestran una tasa de presentación de carcinoma invasor en el mismo sitio de la biopsia del $50 \%$ a 10 años siguientes a la misma; todos de tipo histológico ductal.

2. Aproximadamente la mitad de las pacientes con recurrencia local del CDIS después de manejo conservador muestran carcinoma ductal invasivo.

Con el incremento en la utilización de la mamografía de screening, los países desarrollados han logrado incrementar el número de casos de ca ductal sin invasión. Se informan tasas del $6.6 \%$ de CDIS en biopsias por locali- zación con aguja, y el 30\% de todas las lesiones detectadas mamográficamente son CDIS. Al encontrar lesiones cada vez de menor tamaño, la sobrevida después de tratamiento con mastectomía se aproxima al 100\%. La disección axilar es positiva solo en el 1 a $2 \%$ de los casos, especialmente en aquellas lesiones grandes o palpables y con histología de comedocarcinoma. El tamaño es fundamental y debe establecerse la diferencia entre lesiones macroscópicas y microscópicas, puesto que esto tiene implicaciones pronósticas y terapéuticas.

Se acepta clasificar en dos grupos el carcinoma ductal in situ:

1. No comedocarcinoma

2. Comedocarcinoma

\section{Carcinoma Ductal In Situ de Tipo No Comedocarcinoma}

A su vez se subdivide en estos tipos histológicos:

1. Cribiforme

2. Micropapilar

3. Sólido

Los patrones puros son raros y los dos primeros generalmente se encuentran mezclados; el patrón sólido es el menos común.

E1 método con el cual se hace el diagnóstico (mamografía versus masa palpable) es un indicador importante de la extensión de la lesión y de la probabilidad de invasión, como lo demuestra el siguiente estudio, donde se ve mayor incidencia de microinvasión y enfermedad residual en el espécimen de mastectomía en las pacientes con masa palpable:

\begin{tabular}{lcccc}
$\begin{array}{l}\text { METODO DE } \\
\text { DETECCION }\end{array}$ & $\begin{array}{c}\text { No. } \\
\text { casos }\end{array}$ & CDIS & $\begin{array}{c}\text { \% MICRO } \\
\text { INVASION }\end{array}$ & $\begin{array}{c}\text { Enf. } \\
\text { residual }\end{array}$ \\
\hline Mamografía & 26 & $18(69 \%)$ & $8(31 \%)$ & $11(42 \%)$ \\
Masa palpable & 19 & $11(58 \%)$ & $8(42 \%)$ & $13(68 \%)$ \\
\hline
\end{tabular}

TIPO DE CDIS

\% MICROINVASION

\begin{tabular}{lcc}
\hline Comedocarcinoma & 63 & $(12 / 19)$ \\
Micropapilar & 30 & $(3 / 10)$ \\
Sólido-cribiforme & 0 & \\
\hline
\end{tabular}

Dos terceras partes de los comedocarcinomas tienen microinvasión, solo un tercio de los micropapilares y ninguno de los otros tipos.

La capacidad de la mamografía para detectar lesiones tempranas está en relación directa con la concentración de depósitos de calcio radioopacos en los conductos y conductillos de la lesión principal. Esto a su vez se asocia con detritus celulares y necrosis tisular, que son más frecuentes en el tipo comedo (el cual se caracteriza por necrosis central). Si no hay calcificaciones la lesión no puede ser detectada, ya que la deformidad de tejidos blandos es un hallazgo mamográfico raro en ausencia de infiltración. Sin embargo las calcificaciones no se limitan al tipo comedo; el tipo cribiforme a menudo las presenta y menos comúnmente el tipo micropapilar.

El Bacaf no debe considerarse una prueba confiable para diagnóstico de CDIS: 
1. No obtiene muestra suficiente $\rightarrow$ Falso negativo.

2. Los patrones histológicos no siempre son útiles para diagnóstico de in situ, con la interpretación errónea de probable infiltrante $\rightarrow$ Falso positivo.

\section{¿Cuál es el pronóstico a largo plazo si en una biopsia se detecta un CDIS y la paciente no recibe ningún tratamiento?}

Esta situación ilustra prácticamente todos los conocimientos actuales acerca de la historia natural del CDIS.

Combinando la información proporcionada por series de mastectomía luego de biopsia, con estudios de seguimiento de biopsias sin mastectomía es posible establecer los siguientes principios:

1. En $50 \%$ de los casos con biopsia positiva para CDIS sometidos a mastectomía, no se encuetra enfermedad residual (esto a su vez está relacionado con el tamaño de la lesión y el método de detección, como mencionamos atrás).

2. $25 \%$ de los casos que no se someten a cirugía luego de un diagnóstico de CDIS por biopsia desarrollan un ca invasor en ese mismo sitio.

3. Si partimos de la premisa de que el $50 \%$ de las mujeres con CDIS que se tratan sólamente con biopsia presentan focos residuales de carcinoma ductal in situ, entonces la mitad de dichos focos residuales no evolucionan hacia cáncer clínicamente manifiesto.

4. La mayor parte de los tumores que se desarrollan después de la excisión local planificada y seguimiento mamográfico no son invasores. Antes del advenimiento de la mamografía la mayoría eran invasores y se manifestaban en el transcurso de los 10 años siguientes a la biopsia inicial.

\section{Carcinoma Ductal In Situ de Tipo Comedocarcinoma}

El comedocarcinoma es un tipo histológico de Ca ductal in situ que exhibe necrosis hacia la parte central de la lesión.

Se reconoce la mayor agresividad de esta lesión al compararla con los demás CDIS. Se asocia frecuentemente con:

1. Masa palpable.

2. Mayor extensión de las lesiones.

3. Focos de microinvasión.

4. Ocurrencia ocasional de metástasis ganglionar axilar.

El tratamiento de los comedocarcinomas in situ ha sido generalmente la mastectomía. Se han descrito casos de tratamiento conservador, pero con índices de recurrencia mayores del $50 \%$, con aparición de enfermedad invasiva en el curso de 3 años. La diferencia es notable en comparación con los tipos no comedocarcinoma. Se acepta que tumores menores de $1 \mathrm{~cm}$ son susceptibles de cirugía conservadora más radioterapia; los mayores de 1 $\mathrm{cm}$, mastectomía simple y los mayores de $2,5 \mathrm{~cm}$ mastectomía simple ampliada.

El tamaño per se se asocia con focos de microinvasión, como se ha documentado (Lagios, Smith y Cazter) en lesiones mayores de $2.5 \mathrm{cms}$.

Los resultados preliminares del NSABP 17, aunque no discriminan por tamaño ni tipo histológico, sugieren que la adición de radioterapia a la excisión local amplia disminuye las recurrencias a la mitad, desde un $16.4 \%$ hasta un $7 \%$ en seguimiento a 43 meses. Además se ha observado un menor número de recurrencias invasoras.

\section{Biología Tumoral y enfoque terapéutico}

Durante casi 100 años el tratamiento del carcinoma de mama se basó en la hipótesis Halstediana de biología tumoral del siglo XIX. Era un concepto Mecánico que postulaba que el cáncer se diseminaba en forma ordenada y centrífuga, desde la mama hacia los ganglios regionales y de allí a sitios más distantes.

Se consideraba que el cáncer era una enfermedad local en el momento de su instalación, que los gánglios linfáticos actuaban como barreras para la diseminación local y que la resección de losganglios comprometidos afectaba la supervivencia, lo mismo que la terapia local y regional. Esta hipótesis implicaba tácitamente la idea que el cáncer podía ser controlado siempre y cuando los ganglios fueran resecados en bloque con la mama, y así obtenerse curación. A partir de ese razonamiento se sustentó la implementación de cirugías radicales extendidas con radioterapia adyuvante. La diseminación hematógena no era considerada importante, por lo tanto no se contemplaba ningún tratamiento sistémico.

En la década de 1970-80 Fisher planteó la hipótesis alternativa, basada en experimentos en animales y ensayos clínicos que no demostraban la superioridad de la disección en bloque. Destacó la importancia de la diseminación hematógena y postuló que el carcinoma mamario ya era sistémico en el momento de su instalación. Demostró que los ganglios linfáticos regionales no eran barreras efectivas para contener la diseminación sistémica, sino que más bien eran indicadores de comportamiento biológico y capacidad metastásica, determinada por una compleja interacción entre tumor y huesped. De allí que la resección de los ganglios linfáticos afectados influyera escasamente sobre la supervivencia y que el tipo de terapia locorregional no afectara significativamente la supervivencia. Sugirió que el único factor que podía influír en la historia natural de la enfermedad era un tratamiento sistémico efectivo. Favoreció la aplicación de tratamientos quirúrgicos menos agresivos (con radioterapia complementaria o sin ella), el empleo de quimioterapia y endocrinoterapia coadyuvantes y la realización de disección de los ganglios linfáticos axilares solamente con la finalidad de llevar a cabo una clasificación por estado.

\section{Tratamiento del CDIS}

Las opciones son similares al tratamiento del ca ductal invasor, cuyo manejo se discutirá más adelante.

Existe un mayor riesgo de recurrencia local con el tratamiento conservador, pero muy poca probabilidad de enfermedad metastásica con cualquier opción quirúrgica.

La resección local amplia con márgenes libres más radioterapia es una aceptable alternativa a la mastectomía. Si no se pueden obtener márgenes libres o los resultados cosméticos esperados van a ser pobres, 
debe realizarse mastectomía (ver cuadro de criterios para manejo conservador ). La mayoría de las pacientes con CDIS identificado mamográficamente tienen la posibilidad de elegir cualquiera de las opciones, con tasas de sobrevida a largo plazo excelentes y muy similares. Aunque las tasas de recurrencia local son mayores después de excisión local y radioterapia, y muy altas con excisión local únicamente, la mayoría de las pacientes pueden ser rescatadas con mastectomía en el momento de presentarse la recurrencia local. La disección axilar no se realiza de rutina en la tumorectomía por CDIS en vista de la baja probabilidad de metástasis ganglionar.

Usualmente se adiciona una disección axilar baja a la mastectomía por comedocarcinoma, en la medida que el riesgo de metástasis axilar es mayor y la morbilidad adicional es baja.

Si el CDIS se descubre durante biopsia por proceso benigno y su medida es menor de $0.5 \mathrm{cms}$., no es de tipo comedo y no está asociada a anormalidad mamográfica, ésta paciente tiene clara opción para excisión local amplia más que para mastectomía.

Por el contrario, se prefiere la mastectomía en estas pacientes:

1. Lesiones mayores de $5 \mathrm{cms}$. en su mayor dimensión.

2. Lesiones comedo mayores de $2.5 \mathrm{cms}$.

3. Lesiones palpables.

En estos casos la tasa de recurrencia local luego de tratamiento conservador (incluso seguido de radioterapia) es alta, y la sobrevida será levemente menor que con la mastectomía. Estas pacientes tienen un mayor riesgo de metástasis ganglionar por lo tanto una disección axilar del nivel I debe ser realizada con la mastectomía.

\section{CRITERIOS PARA CIRUGIA CONSERVADORA}

Persigue dos objetivos: control oncológico de la enfermedad y conservar la armonía estética. La localización (p. ej. retroareolar) no es contraindicación.

Toda paciente sometida a cirugía conservadora requiere obligatoriamente:

1. Radioterapia post operatoria para disminuir índices de recaída.

2. Quimioterapia adyuvante cuando cumpla criterios para la misma.

\section{REQUISITOS PARA CIRUGIA CONSERVADORA}

1. Relación mama-tumor favorable

2. Tumor hasta $3 \mathrm{cms}$. Para Comedo hasta $2,5 \mathrm{~cm}$.

3. Facilidad de seguimiento.

4. Deseo de la paciente de conservar su mama.

5. Disponibilidad de radioterapia

6. Que no exista multicentricidad ni componente intracanalicular extenso.

7. Mamografía de base de buena calidad.

8. Que no haya contraindicación para radioterapia (embarazo, colagenopatía).

Las pacientes con bordes de sección comprometidos en la patología definitiva debe ir a mastectomía total con o sin reconstrucción inmediata. En casos especiales po- drá someterse a junta de decisiones la posibilidad de ampliar la resección siempre y cuando cumpla los mismos requisitos para la cirugía conservadora.

\section{ENFERMEDAD DE PAGET IN SITU}

\section{Incidencia}

La enfermedad de Paget de la areola y el pezón afecta aproximadamente el $2 \%$ de las pacientes con carcinoma de mama.

\section{Características Clínicas}

La presentación clínica característica de esta enfermedad, de acuerdo con la descripción de Sir James Paget, corresponde a una lesión eccematosa erosiva del pezón y la areola con descamación, eritema, humedad, ulceración y prurito.

\section{Histología}

Se encuentran células grandes de citiplasma claro en la epidermis, generalmente por arriba de la membrana basal. Dichas células de Paget son PAS positivas y resistentes a la diastasa y son positivas para el antígeno carcinoembrionario ( $\mathrm{ACE}$ ) con las técnicas de la inmunoperoxidasa. Sólamente un $40 \%$ de las muestras se tiñen positivamente para la mucina y en esos casos la tinción es débil. En la enfermedad de Paget extramamaria, un $90 \%$ de las lesiones son intensamente positivas para la mucina.

\section{Diagnóstico}

El diagnóstico se realiza por las características clínicas arriba descritas y mediante biopsia de la lesión, que en la mayor parte de los casos se asocia con un carcinoma invasor. Por consiguiente no es raro encontrar en la mamografía nódulos radiológicamente sospechosos de malignidad. Solo se puede hablar de enfermedad de Paget in situ cuando se descarte un carcinoma infiltrante subyacente.

\section{Diagnóstico Diferencial}

El principal diagnóstico diferencial de la enfermedad de Paget es con el Melanoma Pagetoide Intraepitelial Maligno el cual se simplifica por la demostración de proteina S-100 o inmunoreactividad antigénica específica del melanoma y con el MB45.

\section{Tratamiento}

El tratamiento puede asimilarse al manejo del CDIS, siempre y cuando no exista masa palpable o infiltración. Si ésto se demuestra por patología se trata como un carcinoma infiltrante.

\section{Pronóstico}

La Enfermedad de Paget mamaria no asociada a carcinoma invasor subyacente, aunque poco frecuente, es altamente curable si se le trata en forma adecuada. El índice de curación cuando es manifestación de tumor invasor es esencialmente el mismo que el de la lesión primaria. 